\title{
A Case Where Switching the End Points for Clinical Trial Interpretation Might Be the Right Choice
}

\section{Francesco Perrone, MD, PhD Unità Sperimentazioni Cliniche, Istituto Nazionale per lo Studio e la Cura dei Tumori, Fondazione G.Pascale, IRCCS, Napoli, Italy.}

Massimo Di Maio, MD Oncologia Medica, AO Ordine Mauriziano Ospedale Umberto I, Dipartimento di Oncologia, Università, Torino, Italy.

Lucia Del Mastro, MD UO Sviluppo Terapie Innovative, IRCCS AOU San Martino, Istituto Nazionale per la Ricerca sul Cancro, IST, Genova, Italy.

Corresponding Author: Francesco Perrone, MD, PhD, Unità Sperimentazioni Cliniche, Istituto Nazionale per lo Studio e la Cura dei Tumori, Fondazione G. Pascale, IRCCS, via Mariano Semmola, 80131 Napoli, Italy (f.perrone @istitutotumori.na.it).
The first trial testing the efficacy of prolonging to a total duration of 10 years an aromatase inhibitor (Al) as adjuvant treatment of hormone receptor-positive breast cancer has been reported at the 2016 American Society of Clinical Oncology (ASCO) meeting and published in the New England Journal of Medicine. ${ }^{1}$ In the MA17R study, 1918 postmenopausal patients having previously received 4.5 to 6 years of an $\mathrm{Al}$, preceded in most cases by tamoxifen, were randomized to 5 years of letrozole or placebo. The primary end point was disease-free survival (DFS) defined as the time from randomization to breast cancer recurrence or a new primary breast cancer, whichever came first. Death (either owing or not owing to breast cancer) was not included in the DFS definition, and patients who died without breast cancer recurrence were censored at the date of death. Coherently with study design and eligibility criteria, median age was quite high, 65 years. The high proportion of elderly patients and the low risk of recurrence led to the fact that at the time of analysis there were more deaths (100 in each arm) than breast cancer recurrences or new primaries (67 and 98 with letrozole and placebo, respectively). The primary analysis showed a $4 \%$ advantage in 5 -year DFS for the letrozole arm (95\% vs 91\%) and an HR of 0.66 (95\% $\mathrm{Cl}, 0.48-0.91$ ), with a significant $P$ value of .01. There was no significant difference in overall survival. No other time-to-event analyses were presented at ASCO, and the message coming from the conference and the press coverage was that postmenopausal patients completing 5 years of aromatase inhibitors should or might be offered further 5 years of treatment, notwithstanding prudential comments of the discussants. However, the article reports a posthoc analysis including death within the DFS definition. Such analysis actually shows that the absolute DFS advantage at 5 years is $2 \%$ (from $88 \%$ to $90 \%$ ), the HR is 0.80 (95\% $\mathrm{Cl}, 0.63-1.01)$ or $0.79(95 \% \mathrm{Cl}, 0.63-1.00)$ if adjusted, and the $P$ value is no longer significant, .06 or .05 in the unadjusted and adjusted analysis, respectively (Table).

We argue that the primary end point chosen for the MA17R study has limited clinical relevance, and that the more reasonable interpretation of the trial results should be based on the analysis that includes death as event in the DFS definition. Therefore, the study results should be interpreted as a nonsignificant, very small advantage for patients receiving letrozole. Of course, we recognize that it is methodologically and conceptually wrong to interpret the results of a trial through a secondary end point, ignoring the result of the primary one. This matter is now addressed by the COMPare team ${ }^{2}$ that is systematically checking the top
5 medical journals tracking which trials switched from a primary to a secondary or unplanned outcome in the interpretation of the results. On August 5, 2016, of 67 trials scrutinized only 9 were perfect, 354 outcomes were omitted and 357 new outcomes were added, that were unplanned in the protocol. We presume that in most cases of new added outcomes the unplanned analyses supported a positive interpretation of the trial, whereby planned analyses were negative or not convincing enough. This would not be the case for the MA17R trial, because the primary end point is actually the one that overemphasizes the estimated benefit.

Standardized definitions for efficacy end point in trials of adjuvant treatment of breast cancer have been proposed in 2010 in the so called STEEP system. ${ }^{3}$ Among the proposed definitions, there are none that do not include deaths, at least those deaths owing to breast cancer. Therefore, the DFS definition used in the MA17R analysis is not included in the STEEP system. A DFS analysis including breast cancer deaths, and corresponding to the breast cancer-free interval definition in the STEEP system, was planned a priori as a sensitivity analysis. Obviously, it yielded the same results of the primary analysis without deaths, because breast cancer deaths by definition occur after breast cancer recurrence, and therefore this analysis was useless a priori, being informative only in the rare case of a patient who died of breast cancer but without knowledge of the recurrence date. The real issue, however, is the exclusion of non-breast cancer deaths. This type of event is expected to happen frequently in an old population like the one eligible for this type of trial, and clearly dilutes the clinical relevance of breast cancer specific events. In this setting, death is a relevant competing risk, because it is an alternative outcome that is of equal or even more relevant clinical importance than the primary outcome, and its occurrence alters the probability of the outcome of interest. ${ }^{4}$ The use of a competing risk approach in the statistical analysis has been defined as critical to accurately determining disease risk for elderly individuals, and therefore best inform clinical decision making. ${ }^{5}$ From this point of view, given that death was not included in the definition of the primary end point, a reasonable alternative to modify the primary end point could be performing a competing risk analysis accounting for deaths.

We accept that it might make sense to exclude death in early explanatory trials with new drugs, and in a patient population not particularly at risk of dying for other reasons. But we feel that this choice is questionable in a pragmatic phase 3 trial, with an old 


\begin{tabular}{|c|c|c|c|c|c|c|c|c|}
\hline \multirow[b]{2}{*}{ Characteristic } & \multicolumn{4}{|c|}{ Not Including Death as an Event } & \multicolumn{4}{|c|}{ Including Death as an Event } \\
\hline & $\begin{array}{l}\text { Placebo } \\
(n=959)\end{array}$ & $\begin{array}{l}\text { Letrozole } \\
(n=959)\end{array}$ & $\begin{array}{l}\text { HR } \\
(95 \% \mathrm{Cl})\end{array}$ & $P$ Value & $\begin{array}{l}\text { Placebo } \\
(n=959)\end{array}$ & $\begin{array}{l}\text { Letrozole } \\
(n=959)\end{array}$ & $\begin{array}{l}\text { HR } \\
(95 \% \mathrm{Cl})\end{array}$ & $P$ Value \\
\hline No. of events & 98 & 67 & $\begin{array}{l}0.66 \\
(0.48-0.91)\end{array}$ & .01 (Adjusted) & 100 & 100 & $\begin{array}{l}0.80 \\
(0.63-1.01)\end{array}$ & $\begin{array}{l}.05 \text { (Adjusted) } \\
.06 \text { (Unadjusted) }\end{array}$ \\
\hline 5-Year DFS, \% & 91 & 95 & & & 88 & 90 & & \\
\hline
\end{tabular}

Abbreviations: DFS, disease-free survival, HR, hazard ratio.

drug, where the crucial issue is to decide on the relative value of offering an up to 10-year extension of a treatment already available in clinical practice, to a prevalently old population of patients.

\section{ARTICLE INFORMATION}

Published Online: November 10, 2016.

doi:10.1001/jamaoncol.2016.4896

Conflict of Interest Disclosures: Drs Perrone and Di Maio received honoraria from Novartis and Astra Zeneca. Dr Del Mastro received honoraria from Novartis, Astra Zeneca, and Pfizer.

REFERENCES

1. Goss PE, Ingle JN, Pritchard KI, et al. Extending aromatase-inhibitor adjuvant therapy to 10 years. N Engl J Med. 2016;375(3):209-219.
2. The COMPare Trials Project. Goldacre B Drysdale H, Powell-Smith A, et al. Tracking switched outcomes in clinical trials. http://www.COMPare -trials.org. Accessed October 18, 2016.

3. Hudis CA, Barlow WE, Costantino JP, et al. Proposal for standardized definitions for efficacy end points in adjuvant breast cancer trials: the STEEP system. J Clin Oncol. 2007;25(15): 2127-2132.

4. Gooley TA, Leisenring W, Crowley J, Storer BE. Estimation of failure probabilities in the presence of competing risks: new representations of old estimators. Stat Med. 1999;18(6): 695-706.

5. Berry SD, Ngo L, Samelson EJ, Kiel DP. Competing risk of death: an important consideration in studies of older adults. J Am Geriatr Soc. 2010;58(4):783-787.

Copyright 2016 American Medical Association. All rights reserved. 